\title{
Colectomy Rates in Ulcerative Colitis are Low and Decreasing: 10-year Follow-up Data From the Swiss IBD Cohort Study
}

\author{
Levente Parragi,

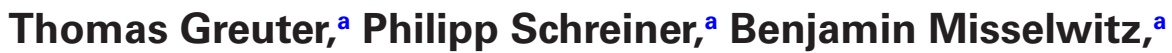 \\ Ekaterina Safroneeva, ${ }^{c}$ A. M. Schoepfer, ${ }^{d}$ Stephan R. Vavricka, ${ }^{\mathrm{e}}$ \\ Gerhard Rogler, ${ }^{a}$ Luc Biedermanna; on behalf of the Swiss IBD \\ Cohort Study Group*
}

\begin{abstract}
${ }^{a}$ Division of Gastroenterology, University Hospital Zurich [USZ] and University of Zurich, Zurich, Switzerland ${ }^{\text {bInstitute }}$ of Social and Preventive Medicine [IUMSP], Lausanne University Hospital, Lausanne, Switzerland 'Institute of Social and Preventive Medicine, University of Bern, Bern, Switzerland Division of Gastroenterology and Hepatology, Centre Hospitalier Universitaire Vaudois [CHUV] and University of Lausanne, Lausanne, Switzerland eDivision of Gastroenterology, Triemli Hospital Zurich, Zurich, Switzerland
\end{abstract}

\begin{abstract}
*Members of the SIBDCS study group: Claudia Anderegg; Peter Bauerfeind; Christoph Beglinger; Stefan Begré; Dominique Belli; José M. Bengoa; Luc Biedermann; Beat Bigler; Janek Binek; Mirjam Blattmann; Stephan Boehm; Jan Borovicka; Christian P. Braegger; Nora Brunner; Patrick Bühr; Bernard Burnand; Emanuel Burri; Sophie Buyse; Matthias Cremer; Dominique H. Criblez; Philippe de Saussure; Lukas Degen; Joakim Delarive; Christopher Doerig; Barbara Dora; Gian Dorta; Mara Egger; Tobias Ehmann; Ali El-Wafa; Matthias Engelmann; Jessica Ezri; Christian Felley; Markus Fliegner; Nicolas Fournier; Montserrat Fraga; Pascal Frei; Remus Frei; Michael Fried; Florian Froehlich; Christian Funk; Raoul Ivano Furlano; Suzanne Gallot-Lavallée; Martin Geyer; Marc Girardin; Delphine Golay; Tanja Grandinetti; Beat Gysi; Horst Haack; Johannes Haarer; Beat Helbling; Peter Hengstler; Denise Herzog; Cyrill Hess; Klaas Heyland; Thomas Hinterleitner; Philippe Hiroz; Claudia Hirschi; Petr Hruz; Rika Iwata; Res Jost; Pascal Juillerat; Vera Kessler Brondolo; Christina Knellwolf; Christoph Knoblauch; Henrik Köhler; Rebekka Koller; Claudia Krieger-Grübel; Gerd Kullak-Ublick; Patrizia Künzler; Markus Landolt; Rupprecht Lange; Frank Serge Lehmann; Andrew Macpherson; Philippe Maerten; Michel H. Maillard; Christine Manser; Michael Manz; Urs Marbet; George Marx; Christoph Matter; Valérie McLin; Rémy Meier; Martina Mendanova; Christa Meyenberger; Pierre Michetti; Benjamin Misselwitz; Darius Moradpour; Bernhard Morell; Patrick Mosler; Christian Mottet; Christoph Müller; Pascal Müller; Beat Müllhaupt; Claudia Münger-Beyeler; Leilla Musso; Andreas Nagy; Michaela Neagu; Cristina Nichita; Jan Niess; Natacha Noël; Andreas Nydegger; Nicole Obialo; Carl Oneta; Cassandra Oropesa; Ueli Peter; Daniel Peternac; Laetitia Marie Petit; Franziska Piccoli-Gfeller; Julia Beatrice Pilz; Valérie Pittet; Nadia Raschle; Ronald Rentsch; Sophie Restellini; Jean-Pierre Richterich; Sylvia Rihs; Marc Alain Ritz; Jocelyn Roduit; Daniela Rogler; Gerhard Rogler; Jean-Benoît Rossel; Markus Sagmeister; Gaby Saner; Bernhard Sauter; Mikael Sawatzki; Michela Schäppi; Michael Scharl; Martin Schelling; Susanne Schibli; Hugo Schlauri; Sybille Schmid Uebelhart; Jean-François Schnegg; Alain Schoepfer; Frank Seibold; Mariam Seirafi; Gian-Marco Semadeni; David Semela; Arne Senning; Marc Sidler; Christiane Sokollik; Johannes Spalinger; Holger Spangenberger; Philippe Stadler; Michael Steuerwald; Alex Straumann; Bigna Straumann-Funk; Michael Sulz; Joël Thorens; Sarah Tiedemann; Radu Tutuian; Stephan Vavricka; Francesco Viani; Jürg Vögtlin; Roland Von Känel; Alain Vonlaufen; Dominique Vouillamoz; Rachel Vulliamy; Jürg Wermuth; Helene Werner; Paul Wiesel; Reiner Wiest; Tina Wylie; Jonas Zeitz; Dorothee Zimmermann
\end{abstract}

Corresponding author: Dr Luc Biedermann, Division of Gastroenterology and Hepatology, University Hospital Zurich, Raemistrasse 100, 8091 Zurich, Switzerland. Tel.: +41-44-255-9519; fax: +41-44-255-9497; email: luc.biedermann@usz.ch 


\begin{abstract}
Objectives: Previous population-based studies in patients with ulcerative colitis [UC] revealed variable colectomy rates and colectomy-associated risk factors. Over the past two decades, a decrease in colectomy rates was observed. We assessed risk factors and colectomy rates over time in UC in the Swiss Inflammatory Bowel Disease Cohort Study [SIBDCS].

Methods: Prospectively collected SIBDCS data, including disease history, baseline characteristics at enrolment, and course of disease, were retrospectively analysed. Cumulative and adjusted annual colectomy rates were calculated.

Results: Among 1245 UC patients analysed [54.6\% male], 114 [9.2\%] underwent colectomy. We observed 5-, 10-, 15-, and 20-year cumulative colectomy rates after diagnosis of $4.1 \%, 6.4 \%$, $10.4 \%$, and $14.4 \%$ of patients, respectively. Male sex (odds ratio [OR] 1.54; $p=0.035$ ), pancolitis at diagnosis [OR $=2.16 ; p=0.005$ ], younger age at diagnosis [OR 0.89 per 5 years of age; $p=0.006$ ] and presence of extraintestinal manifestations [EIM] [OR 2.30; $p<0.001$ ] were risk factors for undergoing colectomy. We did not observe a significant protective effect of smoking on colectomy risk [OR $0.64 ; p=0.106$ ]. The majority of colectomies were performed within first 10 years of disease onset, with a rapidly decreasing colectomy rate after 15 years. In patients diagnosed after 2003, colectomy was performed much earlier during and individual's disease course. Nevertheless, we found a significantly decreasing trend in yearly colectomy rates over time after 2005.

Conclusions: Crude and adjusted colectomy rates in Swiss UC patients were lower than those reported previously in the literature, and decreased over time.
\end{abstract}

Key Words: Ulcerative colitis; colectomy rates; risk factors; cohort study

\section{Introduction}

UC refers to a chronic inflammatory condition of yet insufficiently understood aetiology, affecting the mucosal layer of the colon. Colectomy, hospitalisation due to severe flares, and colorectal cancer are amongst the most important adverse outcomes in patients with UC. ${ }^{1-5}$ Main indications for colectomy in UC patients can typically be distinguished into emergent colectomy, with fulminant or refractory colitis, and subacute to chronic complications constituting indication for surgery in the setting of elective colectomy, with colonic dysplasia or cancer and intolerance to medical treatment as the main underlying indications necessitating surgery. ${ }^{6,7}$

In previous studies, rates of colectomy in UC patients were revealed to be highly variable over time and geographical location. In a retrospective population-based series from Sweden in 1990, high cumulative colectomy rates of $20 \%$ after 5 years, $28 \%$ after 10 years, and $45 \%$ after 25 years were described. ${ }^{8}$ Similar results were reported in a Danish cohort from Copenhagen in 1994, with $24 \%$ of UC patients undergoing colectomy 10 years after diagnosis. ${ }^{9}$ More recent studies, however, revealed considerably lower colectomy rates. The European Collaborative Study Group of Inflammatory Bowel Disease [EC-IBD] reported a cumulative 10 -year colectomy rate of $8.7 \%$. Intriguingly, a substantial difference in colectomy rates was discovered in northern [10.4\%] as compared with southern European centres [3.9\%]. ${ }^{10}$ Investigations by the Inflammatory Bowel South-Eastern Norway [IBSEN] Study Group determined a 1-year colectomy rate of $4 \% .{ }^{11}$ After 5 years, $7.5 \%$ of patients underwent colectomy; the majority among them [almost three out of four; $71 \%$ ] within the first 2 years following diagnosis. ${ }^{12}$ After 10 years of follow-up, the cumulative colectomy rate was $9.8 \%$ in this cohort. ${ }^{13}$ Similarly, a Canadian populationbased study revealed 5-, 10-, and 20-year overall colectomy rates of $7.5 \%, 10.4 \%$, and $14.8 \%$, respectively. ${ }^{14}$ In addition, researchers from the Mount Sinai Ulcerative Colitis Surveillance Database, including 561 patients, identified a colectomy rate of $17.3 \%$ over a median follow-up of 21.4 years. $^{2}$

These considerably lower colectomy rates compared with the Scandinavian population-based studies ${ }^{8,9}$ are most likely due to a multitude of reasons, including variations in diagnostic accuracy and referral bias, as well as substantial improvements in therapeutic opportunities over time. Regarding the latter, Kaplan et al. described steadily decreasing elective but stable emergent colectomy rates between 1997 to 2009, possibly due to an increase in use of immunomodulators and anti-tumour necrosis factor $[\mathrm{TNF}] .^{7}$ In contrast, Jess et al. observed no significant change in surgery rates in Denmark from 1962 to 2005 in UC patients, reporting a fairly stable longterm prognosis over the past five decades. ${ }^{15}$

Several risk factors have been associated with future need of colectomy. For instance, tobacco smoking has been described to exert a protective effect, with smokers being less likely to develop UC compared with non-smokers. ${ }^{16-18}$ Further, several studies indicated active smoking to be associated with milder course of UC, including lower colectomy rates in some $\mathrm{e}^{19,20}$ but not all ${ }^{21}$ studies. Numerous studies identified male $\operatorname{sex}^{13,14,22,23}$ and disease extent at diagnosis as major risk factors. ${ }^{7-12}$ Patients with extensive colitis at diagnosis have a higher probability of colectomy compared with patients with proctitis and left-sided colitis, with an increasing risk over time in the case of disease progression from proctitis or left-sided colitis to extensive colitis during follow-up. . $^{8,10,13}$

Extraintestinal manifestations [EIM] may worsen the course of disease and therefore be associated with higher risk for complications. For instance, the risk of developing dysplasia or colorectal cancer is substantially increased in UC patients with concomitant primary sclerosing cholangitis [PSC], consecutively affecting colectomy rates. ${ }^{3,24}$

In a systematic review and meta-analysis including 71 studies, no difference in need for surgery was identified amongst those patients 
with versus without family history of UC. ${ }^{25}$ Likewise, no difference in the course of disease, including need for surgery, was observed between familial and sporadic inflammatory bowel disease [IBD] cases in a population-based study in Norway. ${ }^{26}$

In view of the currently limited and partially conflicting available literature on this topic, we aimed to investigate the cumulative rates of colectomy and potential changes thereof over time, as well as associated risk factors in UC patients in the Swiss Inflammatory Bowel Disease Cohort Study [SIBDCS].

\section{Material and Methods}

\subsection{Patients}

Starting in 2006, patients with an established diagnosis of IBD from all regions of Switzerland were included in the nationwide Swiss Inflammatory Bowel Disease Cohort Study $[\text { SIBDCS }]^{27}$ that is financially supported by the Swiss National Science Foundation [SNSF] and approved by the local ethics committees [EK-1316]. All patients provided written informed consent. Recruitment of patients is carried out in university hospitals, large non-university hospitals and private practices across Switzerland.

Patients with Crohn's disease [CD], UC or colitis unclassified [CU] are included in the cohort if diagnosis was established at least 4 months before inclusion. At the time of inclusion, data acquisition focuses on clinical, socioeconomic, and psychosocial data. Patients then undergo a thorough clinical and laboratory assessment. Treating IBD physicians complete detailed questionnaires focusing on physician-reported outcomes, whereas patient-reported outcome questionnaires evaluate quality of life, social impairment, and symptoms. Follow-up occurs once a year and additionally in case of unscheduled events.

All patients within the SIBDCS with a diagnosis of UC established by a gastroenterologist [including patients having received their diagnosis of UC or having undergone colectomy before the initiation of the SIBDCS] were included in our analysis. We conducted an analysis of the prospectively collected data during SIBDCS follow-up, including disease history characteristics at enrolment in the cohort. Colectomy rates and time of diagnosis in SIBDCS patients before inclusion in the cohort could be assessed through enrolment and follow-up questionnaire data. ${ }^{27}$

Non-smokers were defined as patients who had never smoked, either at diagnosis or during course of disease and follow-up in the SIBDCS; whereas ongoing smokers were defined as either patients who had been smoking at diagnosis of their UC and at least for one consecutive follow-up visit, or as patients not smoking at diagnosis but reporting smoking during follow-up at least once in the annual patient questionnaire. Smoking status is assessed at inclusion and also longitudinally in the annual patient as well as physician questionnaires of the SIBDCS. As smoking status enquired by the physician may be under-reported, we considered self-reporting in the patient questionnaire for our analysis.

\subsection{Statistical analysis}

Statistical analyses were carried out using the STATA software [v. 14.2, StataCorp, College Station, TX, USA] and the R software [v 3.23, R Foundation for Statistical Computing, Vienna, Austria].

Continuous data distribution was assessed using Normal QQ-plots. Normally-distributed continuous variables were summarised as mean, standard deviation, and range. Non-Gaussian data were summarised as median, interquartile range [IQR], and range. Differences in means between two groups for normally distributed data were assessed using Student's t-test, and differences in distribution for non-Normally distributed data were assessed using the Mann-Whitney Wilcoxon rank sum test. In case of more than two groups, analysis of variance [ANOVA], [respectively Kruskall-Wallis test], has been used.

Categorical data were summarised as raw frequencies and relative percentages. Differences in categorical data distribution between two or several groups have been assessed using the chi square test, or Fisher's exact test in case of insufficient sample size.

Cumulative rates of colectomy were analysed using survival analysis, including specific techniques to deal with left- and intervalcensored data. Kaplan-Meier curves were computed to analyse time to first occurrence of colectomy and to derive, for example, 10-year colectomy rates of complications, with $95 \%$ confidence intervals [CI]. The log-rank test was used to compare several survival curves, according to the different risk factors.

Multivariate logistic regression was used to assess association between colectomy rates and different risk factors. Crude yearly colectomy rates were computed with $95 \%$ confidence intervals. Adjusted yearly rates of colectomy were computed using multilevel mixed-effects logistic regression, by forcing a linear effect of calendar year on the logistic level. Adjustment has been made according to different risk factors.

\section{Results}

\subsection{Baseline characteristics of patients and overall colectomy rates}

We included a total of 1245 SIBDCS patients [680 male, 54.6\%] with UC in our analysis, with a median disease duration of 11 years and median age at diagnosis of 31 years. Out of these 1245 patients, 114 underwent colectomy, with an overall 5-, 10-, 15-, and 20-year cumulative colectomy rate after diagnosis of $4.1 \%$ [CI: 3.09-5.41\%], 6.3\% [CI: $5.00-8.00 \%$ ], $10.5 \%$ [CI: $8.55-12.95 \%$ ], and $14.8 \%$ [CI: $12.06-18.02 \%]$, respectively. The majority of colectomies $[73.7 \%$, 84 out of 114] were performed after inclusion in the SIBDCS. The indication for colectomy was refractory disease in the vast majority of cases, with colorectal cancer [4.8\%] and dysplasia [7.1\%] in the remaining cases [Tables 1,2]. At diagnosis $37.5 \%$ of our patients had pancolitis [E3], 32.1\% left-sided UC [E2], and 20.4\% ulcerative proctitis [E1] at diagnosis according to the Montreal classification ${ }^{28 ;}$ whereas at the last assessment before colectomy including 84 patients who received colectomy during follow-up, $46.4 \%$ had pancolitis [E3], 29.8\% left-sided UC [E2], and 5.9\% ulcerative proctitis [E1]. For the remaining 30 patients the last assessment before colectomy was not available, due to the fact that they underwent colectomy before inclusion in the cohort.

Colectomised patients [114] had an extensive medical history of treatment with thiopurines [74.5\%], any anti-TNF [48.2\%], methotrexate $[21.1 \%$ ], cyclosporine [29.8\%], and tacrolimus [14\%].

\subsection{Risk factors for colectomy}

In a univariate analysis, we found male sex to be significantly associated with colectomy, with almost two-thirds of patients [64\%] undergoing colectomy being male [female: $\mathrm{OR}=0.65 ; p=0.035$ ]. Pancolitis at diagnosis was also a highly significant risk factor for colectomy, with a more than $20 \%$ higher proportion of patients with pancolitis among those UC patients undergoing colectomy as compared with their counterparts without colectomy during followup in the SIBDCS [left-sided colitis: OR 0.39; $p<0.002$; proctitis: OR $0.25 ; p<0.001]$. Furthermore, we found younger age at diagnosis to be associated with higher colectomy rates $[\mathrm{OR}=0.89$ per 5 years of age; $p=0.006]$. In addition, there was a non-significant 
association of positive family history of IBD with higher colectomy rates $[\mathrm{OR}=1.69 ; p=0.060$; Tables 1 and 2$]$.

In multivariate analyses, pancolitis at diagnosis $[\mathrm{OR}=2.16$; $p=0.005]$ and disease duration $[\mathrm{OR}=1.04 ; p=0.001]$ were revealed to be independently associated with colectomy, whereas sex or age at diagnosis were not [Table 2]. A statistically significant linear diminishing trend with year on the logistic level for colectomy rates was found (beta coefficient $=-0.210[-0.323--0.097] ; p<0.001$ ) when adjusting for gender, age at diagnosis, disease duration, and previous use of anti-TNF agent.

\subsubsection{EIM and colectomy}

We found a significantly higher fraction of patients suffering from concomitant extraintestinal manifestations [EIM] at any time in their entire history of disease, with $59.6 \%$ in patients with as compared with $39.1 \%$ in those without colectomy [OR $=2.30 ; p<0.001]$. This increase in risk was observed for EIM overall and several individual EIM [Table 1].

\subsubsection{Smoking and colectomy}

At the last assessment before colectomy, $89.3 \%$ of the patients were non-smokers [Table 3]. Smoking did not have a significant protective effect on the risk of colectomy overall in the SIBDC; however, a potential protection might have occurred in smoking male patients $[\mathrm{OR}=0.64 ; p=0.106]$. Likewise, considering only the most recent smoking status [i.e. at latest follow-up or at follow-up preceding surgery] we did not observe any significant protective effects in the whole study population, nor in the subgroups of male or female patients.

Table 1. Baseline patient characteristics.

\begin{tabular}{|c|c|c|c|c|}
\hline & No colectomy & Colectomy & All ulcerative colitis patients & $p$-Value \\
\hline Number of patients & $1131[90.8]$ & $114[9.2]$ & $1245[100.0]$ & - \\
\hline \multicolumn{5}{|l|}{ Gender } \\
\hline Male & $607[53.7]$ & $73[64.0]$ & $680[54.6]$ & \\
\hline Female & $524[46.3]$ & $41[36.0]$ & $565[45.4]$ & 0.034 \\
\hline Age at diagnosis [y] & 31,24 to 41 & 28,23 to 35 & 31,23 to 41 & 0.006 \\
\hline [median, IQR, range] & 3 to 83 & 11 to 75 & 3 to 83 & \\
\hline Age at enrollment $[\mathrm{y}]$ & 41,31 to 52 & 39,31 to 50 & 41,31 to 52 & 0.344 \\
\hline [median, IQR, range] & 16 to 85 & 18 to 76 & 16 to 85 & \\
\hline Age at latest follow-up [y] & 46,36 to 57 & 46,37 to 57 & 46,36 to 57 & 0.848 \\
\hline [median, IQR, range] & 17 to 89 & 22 to 82 & 17 to 89 & \\
\hline Disease duration $[y]$ & 11,6 to 18 & 15,9 to 22 & 11,6 to 18 & $<0.001$ \\
\hline [median, IQR, range] & 0 to 50 & 2 to 44 & 0 to 50 & \\
\hline Body mass index $\left[\mathrm{kg} / \mathrm{m}^{2}\right]$ & $23.7,21.5$ to 26.4 & $23.4,20.8$ to 26.4 & $23.6,21.5$ to 26.4 & 0.392 \\
\hline [median, IQR, range] & 15.1 to 62.5 & 16.0 to 48.8 & 15.1 to 62.5 & \\
\hline \multicolumn{5}{|c|}{ Family history of inflammatory bowel disease } \\
\hline None & $885[78.3]$ & $90[79.0]$ & $975[78.3]$ & \\
\hline Yes & 112 [9.9] & 19 [16.7] & $131[10.5]$ & \\
\hline Unknown & $134[11.8]$ & $5[4.4]$ & $139[11.2]$ & 0.008 \\
\hline \multicolumn{5}{|l|}{ Disease location at diagnosis } \\
\hline Pancolitis & $402[35.5]$ & $65[57.0]$ & $467[37.5]$ & \\
\hline Left-sided colitis & 375 [33.2] & 24 [21.1] & 399 [32.1] & \\
\hline Proctitis & $244[21.6]$ & $10[8.8]$ & $254[20.4]$ & \\
\hline Unknown/unclear & $110[9.7]$ & 15 [13.2] & $125[10.0]$ & $<0.001$ \\
\hline \multicolumn{5}{|c|}{ History of extraintestinal manifestation ever in course of disease } \\
\hline None & $689[60.9]$ & $46[40.4]$ & $735[59.0]$ & \\
\hline Yes & $442[39.1]$ & $68[59.6]$ & $510[41.0]$ & $<0.001$ \\
\hline Arthralgia & $362[32.0]$ & $52[45.6]$ & $414[33.3]$ & 0.003 \\
\hline Uveitis/iritis & $64[5.7]$ & 11 [9.6] & $75[6.0]$ & 0.088 \\
\hline Pyoderma gangrenosum & $18[1.6]$ & $4[3.5]$ & $22[1.8]$ & 0.135 \\
\hline Erythema nodosum & $37[3.3]$ & $8[7.0]$ & $45[3.6]$ & 0.041 \\
\hline Aphtous/oral ulcers & $56[5.0]$ & $6[5.2]$ & $62[5.0]$ & 0.884 \\
\hline Ankylosing spondylitis & $35[3.1]$ & $6[5.2]$ & $41[3.3]$ & 0.216 \\
\hline Primary sclerosing cholangitis & $33[2.9]$ & 18 [15.8] & $51[4.1]$ & $<0.001$ \\
\hline \multicolumn{5}{|c|}{ History of treatment ['ever treated with'] } \\
\hline 5-aminosalicylic acid & $1086[96.0]$ & $103[90.4]$ & $1189[95.5]$ & 0.005 \\
\hline Steroids & $884[78.2]$ & $105[92.1]$ & 989 [79.4] & $<0.001$ \\
\hline Thiopurines & $649[57.4]$ & $85[74.5]$ & $734[59.0]$ & $<0.001$ \\
\hline Methotrexate & $87[7.7]$ & $24[21.1]$ & $111[8.9]$ & $<0.001$ \\
\hline Infliximab & $330[29.2]$ & $51[44.7]$ & $381[30.6]$ & 0.001 \\
\hline Adalimumab & $91[8.0]$ & $16[14.0]$ & $107[8.6]$ & 0.030 \\
\hline Certolizumab & $4[0.4]$ & $4[3.5]$ & $8[0.6]$ & 0.003 \\
\hline Any anti-tumour necrosis factor & $352[31.1]$ & $55[48.2]$ & $407[32.7]$ & $<0.001$ \\
\hline Cyclosporine & $63[5.6]$ & $34[29.8]$ & $97[7.8]$ & $<0.001$ \\
\hline Tacrolimus & $38[3.4]$ & $16[14.0]$ & $54[4.3]$ & $<0.001$ \\
\hline
\end{tabular}

$\mathrm{IQR}$, interquartile range; y, years. 
Table 2. Multivariate analysis of baseline characteristics.

\begin{tabular}{|c|c|c|c|c|}
\hline & \multicolumn{2}{|l|}{ Univariate analysis } & \multicolumn{2}{|l|}{ Multivariate analysis $^{a}$} \\
\hline & OR $[95 \% \mathrm{CI}]$ & $p$-Value & OR $[95 \% \mathrm{CI}]$ & $p$-Value \\
\hline Gender [ref: male] & $0.651[0.436-0.971]$ & 0.035 & & \\
\hline Age at diagnosis [per 5 years] & $0.894[0.825-0.968]$ & 0.006 & & \\
\hline Age at enrollment [per 5 years] & $0.961[0.898-1.028]$ & 0.247 & & \\
\hline Age at latest follow-up [per 5 years] & $0.986[0.923-1.053]$ & 0.676 & & \\
\hline Disease duration [per 5 years] & 1.177 [1.072-1.293] & 0.001 & $1.223[1.082-1.383]$ & 0.001 \\
\hline Body mass index [per point] & $0.990[0.946-1.036]$ & 0.659 & & \\
\hline Family history of inflammatory bowel disease [ref: no family history] & $1.668[0.979-2.841]$ & 0.060 & & \\
\hline \multicolumn{5}{|l|}{ Initial ulcerative colitis location [ref: pancolitis] } \\
\hline Left-sided colitis & $0.396[0.243-0.645]$ & $<0.001$ & $0.463[0.242-0.788]$ & 0.005 \\
\hline Proctitis & $0.253[0.128-0.503]$ & $<0.001$ & $0.334[0.163-0.688]$ & 0.003 \\
\hline Smoking status at dianosis. [ref: non-smoker] & $0.643[0.376-1.098]$ & 0.106 & & \\
\hline History of EIM [ref: no EIM recorded] & $2.304[1.556-3.413]$ & $<0.001$ & & \\
\hline \multicolumn{5}{|l|}{ Therapy history [ref: never treated] } \\
\hline 5-amino salicylic acid & $0.388[0.195-0.773]$ & 0.007 & & \\
\hline Steroids & $3.260[1.627-6.533]$ & 0.001 & & \\
\hline Thiopurines & $2.177[1.405-3.372]$ & $<0.001$ & & \\
\hline Methotrexate & $3.200[1.940-5.279]$ & $<0.001$ & $2.927[1.598-5.360]$ & 0.001 \\
\hline Anti-tumour necrosis factor & $2.063[1.399-3.042]$ & $<0.001$ & & \\
\hline Cyclosporine & $7.205[4.481-11.585]$ & $<0.001$ & $9.016[5.167-15.732]$ & $<0.001$ \\
\hline Tacrolimus & $4.696[2.527-8.726]$ & $<0.001$ & & \\
\hline
\end{tabular}

OR, odds ratio; CI, confidence interval; EIM, extraintestinal manifestations.

${ }^{a}$ Multivariate analysis of baseline patient characteristics according to Table 1. *Multivariate logistic regression analysis was carried out by including all univariate factors with $p$-value $<0.200$, removing non-significant factors one by one until all factors remain significant. Model consistency was then tested by reintroducing excluded factors one by one until final model selection.

Table 3. Details of baseline characteristics of colectomy patients.

\begin{tabular}{lc}
\hline Characteristic & Number [\%] \\
Smoking status at colectomy & \\
Non-smoker & $75[89.3 \%]$ \\
Smoker & $7[8.3 \%]$ \\
Unknown & $2[2.4 \%]$ \\
Disease location at colectomy & \\
Pancolitis & $39[46.4 \%]$ \\
Left-sided colitis & $25[29.8 \%]$ \\
Proctitis & $5[5.9 \%]$ \\
Unknown/unclear & $15[17.9 \%]$ \\
Complications preceding colectomy & \\
Colorectal cancer & $4[4.8 \%]$ \\
Colon dysplasia & $6[7.1 \%]$ \\
Therapy history preceding colectomy & \\
5-aminosalicylic acid & $76[90.5 \%]$ \\
Steroids & $81[96.4 \%]$ \\
Thiopurines & $65[77.4 \%]$ \\
Methotrexate & $25[29.8 \%]$ \\
Infliximab & $44[52.4 \%]$ \\
Adalimumab & $20[23.8 \%]$ \\
Certolizumab & $4[4.7 \%]$ \\
Any anti-tumour necrosis factor & $48[57.8 \%]$ \\
Cyclosporine & $25[29.8 \%]$ \\
Tacrolimus & $13[15.5 \%]$ \\
\hline
\end{tabular}

Details on patients undergoing colectomy during follow-up [84 patients in total].

\subsection{Disease duration and colectomy}

Overall colectomy rates were highest in the 10-20 years following UC diagnosis [Table 4], and numerically the majority of colectomies were performed within 10 years after UC diagnosis. The highest
Table 4. Time trends: colectomy rates according to decades.

\begin{tabular}{lrrrr}
\hline $\begin{array}{l}\text { Disease } \\
\text { duration }\end{array}$ & Person-time & $\begin{array}{l}\text { Number of } \\
\text { colectomies }\end{array}$ & $\begin{array}{l}\text { Rate of } \\
\text { colectomy }\end{array}$ & $\begin{array}{l}\text { 95\% confidence } \\
\text { interval }\end{array}$ \\
\hline 0-10 years & 9737.24 & 67 & 0.006881 & $0.00542-0.00874$ \\
$>10-20$ years & 4255.19 & 39 & 0.009165 & $0.00669-0.01254$ \\
$>20-30$ years & 1470.01 & 7 & 0.004762 & $0.00227-0.00999$ \\
$>30-40$ years & 409.98 & 1 & 0.002439 & $0.00034-0.01732$ \\
\hline
\end{tabular}

Rate of colectomy according to decades, analysed patients in time and numbers of colectomies performed.

fraction of patients undergoing colectomy had their diagnosis before 1997 [Table 5].

In view of the fact that crude numbers of colectomy have to be interpreted with caution [increase in overall risk in patients with longer follow-up], we subsequently analysed our data in addition in a time-to-event fashion with the survival approach, revealing a colectomy-free survival of $0.936,0.856$, and 0.816 after 10,20 , and 30 years of disease duration, respectively [Figure 1]. As shown by the smoothed hazard function [depicting the instantaneous colectomy risk at an exact time, conditional on survival until then], we found the risk of colectomy to initially increase after 10 years, reaching a peak after about 15 years of disease duration, and to subsequently decrease to considerably lower risk rates [Figure 2, Table 4].

\subsection{Time trends in colectomy rates: [evolution of colectomy rates over time]}

We observed a decrease in colectomy rates over time, with evident nominal decrease in absolute numbers of annual colectomies performed [as well as the incident rate of colectomy] after 2010 
Table 5. Time trends: disease duration and colectomy.

\begin{tabular}{llll}
\hline & No colectomy & Colectomy & All ulcerative colitis patients \\
\hline Number of patients & $1131[90.8]$ & $114[9.2]$ & $1245[100.0]$ \\
Disease duration [years] & $11,6-18$ & $15,9-22$ & $11,6-18$ \\
[median, IQR, range] & 0 to 50 & 2 to 44 & 0 to 50 \\
Diagnosis year & & & $332[26.7]$ \\
$\quad 1996$ or before & $287[25.4]$ & $45[39.5]$ & $310[24.9]$ \\
19972003 & $279[24.7]$ & $31[27.2]$ & $346[27.8]$ \\
20042008 & $314[27.8]$ & $32[28.1]$ & $257[20.6]$ \\
2009 or later & $251[22.2]$ & $6[5.3]$ & $<.001$ \\
\hline
\end{tabular}

Collectomy rates according to disease duration in years [y] and year of diagnosis.

$\mathrm{IQR}$, interquartile range.

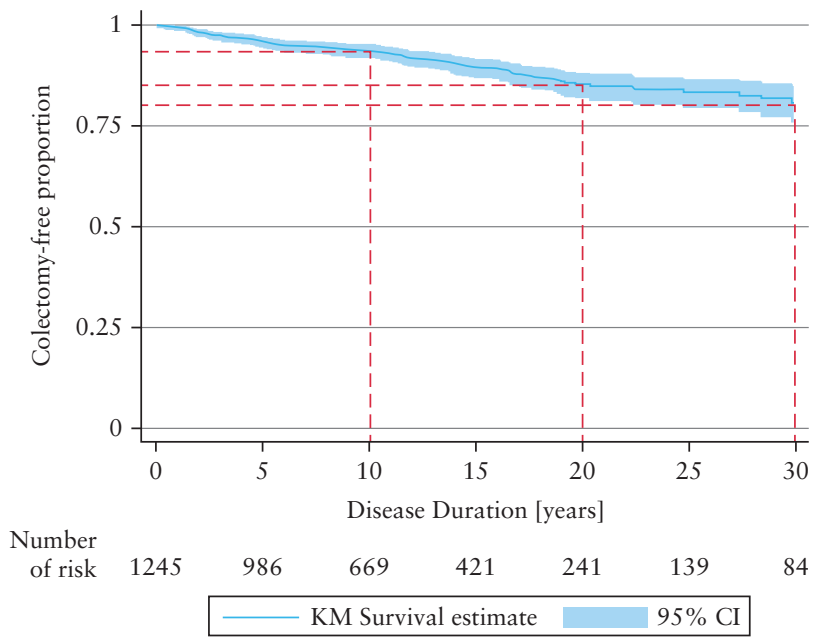

Figure 1. Survival rate of colectomy-free proportion across 30 years of disease duration. The observed survival rate of colectomy-free proportion over time according to disease duration is depicted, including numbers of patients at risk as illustrated in a Kaplan-Meier survival estimate and a 95\% confidence interval $[\mathrm{Cl}=$ confidence interval $]$.

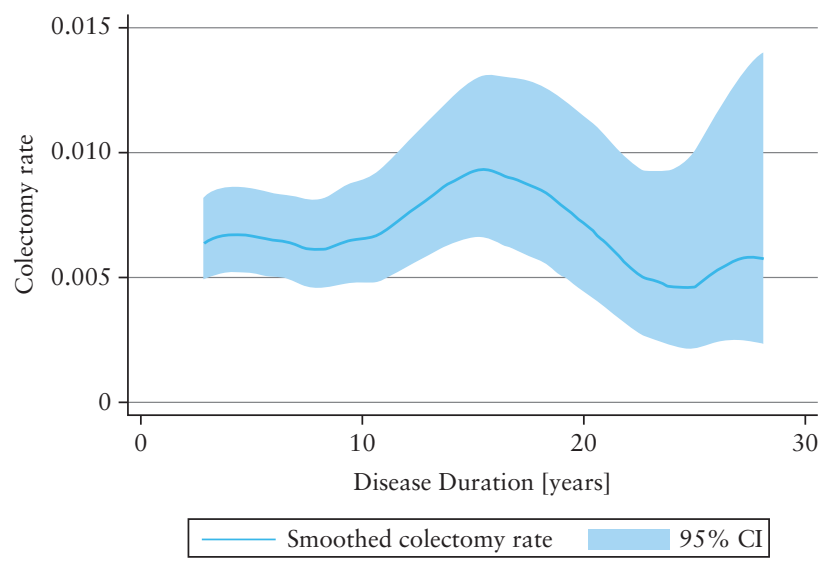

Figure 2. Colectomy rate according to disease duration. The smoothed hazard estimate of the colectomy incidence rate according to duration of disease is shown with a smoothed hazard function and $95 \%$ confidence interval. After 15 years of disease duration, a decrease in hazard rate can be observed $[\mathrm{Cl}=$ confidence interval].

[Figure 3]. In an unadjusted crude prediction [forcing linear effect of year on the logistic level] of yearly colectomy rates, we found a significantly decreasing linear trend $[p=0.037]$ already after 2005
[Figure 3]. For this entire period of time, when adjusting for disease duration, age at diagnosis, gender, and previous use of antiTNF, a statistically highly significant linear diminishing trend with year on the logistic level for colectomy rates was found (beta coefficient $=-0.210[-0.323--0.097] ; p<0.001)$. Importantly, by far the lowest colectomy rates observed in our follow-up time were detected in the last 3 years of observation, i.e. 2013-2015. The observed survival rate without colectomy, analysed in a time-to-event fashion and stratified by patients diagnosed before and after 2003 [with 2003 as the median year of diagnosis among our cohort patients; i.e. half of the patients were diagnosed before and after 2003], indicated that colectomy was performed significantly earlier during the individual course of disease in recently diagnosed patients [significant difference in survival curves between patients diagnosed before and after 2003 in log-rank test, $p=0.006$ ]. Cumulative proportions of colectomy at 2, 5, or 10 years after diagnosis were roughly twice as high in the more recently diagnosed subgroup of cohort patients, with $2.50 \%, 5.96 \%$, and $8.64 \%$ versus $1.09 \%, 2.65 \%$, and $4.71 \%$ in patients diagnosed after and before 2003, respectively.

\section{Discussion}

In this study we investigated the risk of colectomy in a large prospective cohort of UC patients over a considerable, prospective, long-term follow-up with a median disease duration of 11 years [with a range up to 50 years, IQR 6 to 18 years]. We would like to emphasise the following key findings. First, we found overall colectomy rates in our cohort at the lower end of or even lower than those described in previous literature. Second, we identified significantly decreasing colectomy rates in Swiss UC patients within recent years. Third, the risk of colectomy according to disease duration follows a bell-shaped curve, with a monotonous increase in colectomy rates during the first 15 years after diagnosis, followed by a subsequent decrease in colectomy risk.

The overall reduction of colectomy rates represents an encouraging signal for patients with recently diagnosed UC as well as those with a disease duration of over 20 years.

Our results reveal considerably lower cumulative colectomy rates of $4.1 \%, 6.3 \%, 10.5 \%$. and $14.8 \%$ after $5-, 10-, 15-$. and 20 years, respectively, as compared with historical studies [20.0-23.7\%, 28.0$29.9 \%$, and $32.4-45.0 \%$ after 10,15 , and 20 years, respectively]. ${ }^{8,9}$ In addition to that, our observed cumulative rates also appear in line with ${ }^{2,13,14}$ or slightly lower than those observed in more recent investigations [ $7.6 \%$ and $9.8 \%$ after 5 and 10 years, ${ }^{13}$ and $7.5 \%$ and $10.4 \%{ }^{14}$ after 5 and 10 years, respectively].

In terms of risk factors for colectomy, our study confirmed male $\operatorname{sex}^{13,14,22,23}$ [almost twice as many colectomies were performed on male patients], lower median age at diagnosis [in accordance with ${ }^{14}$ 


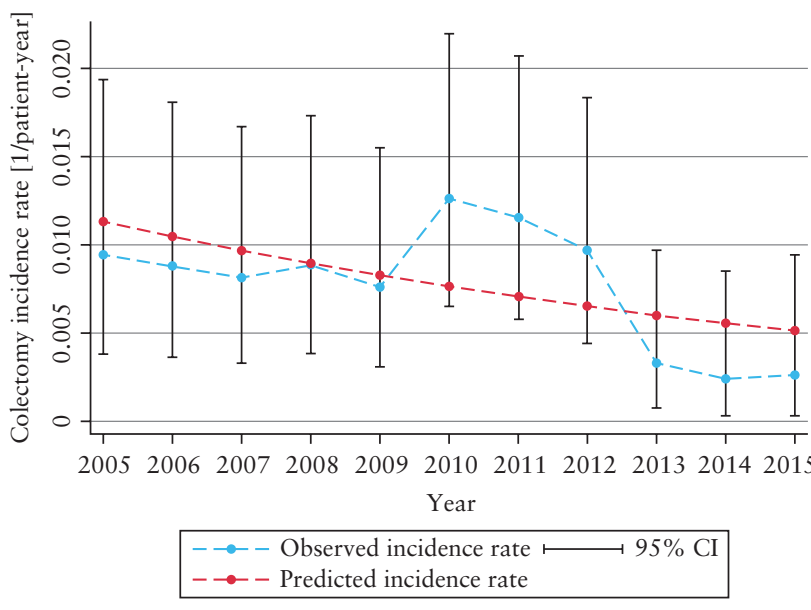

Figure 3. Predicted and observed incidence rates of colectomy over 20052015. The observed incidence rate of colectomy over time is depicted in conjunction with a crude, unadjusted predicted yearly rate $[\mathrm{Cl}=$ confidence interval].

and in contrast to ${ }^{10}$ previous investigations], positive family history of UC [in contrast to a previous meta-analysis from $2014^{25}$ ], and pancolitis [more than half of all patients undergoing colectomy suffered from pancolitis at diagnosis, in line with a meta-analysis ${ }^{23}$ ] whereas higher age was protective [similar to previous studies]. ${ }^{13,29}$

Our study showed individual EIM to be significantly associated with colectomy; in particular arthralgia was present in almost half of all colectomised patients. This is in line with population-based studies from France in children, ${ }^{30}$ adults, ${ }^{31}$ or both. ${ }^{32}$ Interestingly, in the the latter, EIM were a rather strong risk factor for a composite endpoint of complications [including use of immunosuppressants or biologics, or need of colectomy].

It has been repetitively described that tobacco smoking is protective against UC and associated with a milder course of disease, ${ }^{16-18}$ but a potential protective effect on the risk of colectomy remains dubious and unproven. ${ }^{10,33,34}$ Likewise, our results do not support a negative effect of smoking cessation or non-smoking on the overall risk of colectomy. Our results thus provide further rationale to encourage smoking cessation even in severe UC cases and to ultimately discard still widely prevalent concerns among both physicians and patients about this step.

Regarding evolution of colectomy rates over time, our findings indicate a clear decrease. However, if necessary, colectomy is increasingly performed early in the course of disease as reported in previous studies. ${ }^{12-14,35}$ Our finding of a significantly earlier performance of colectomy in recently diagnosed patients may point to an increased awareness and prompter diagnosis, as well as an increasingly early administration of potent and/or novel medical treatment after diagnosis, i.e. following a rapid step-up approach. The latter potentially translates into a benefit for our patients, with shorter period of time lost with futile medical therapies. A tendency towards a more aggressive treatment approach early in the course of disease may suit as an explanation for both the decrease in overall rates of colectomy in UC patients on the one hand, and an increasingly short interval between diagnosis and colectomy on the other. In view of a more rapidly enabled treatment escalation, it may be speculated that the peak of colectomy risk after diagnosis [Figure 2] may occur even earlier after diagnosis in the future, whereas the overall risk of colectomy may continuously decrease. Regarding the latter, an earlier and more frequent use of immunomodulators and the introduction of biologics has been shown to reduce the requirement for surgery in the long term. ${ }^{1}$

Our study has several limitations. Prospective data acquisition in the cohort started only in 2006, so antecedent data are retrospective. Thus, we cannot exclude a recruitment bias affecting colectomy rates before 2006. For instance, if patients underwent colectomy before prospective data acquisition, these patients might not have been included in the cohort at all, potentially leading to a relevant underestimation of the colectomy rate before 2006. Yet, in clinical practice in Switzerland, UC patients with a very severe disease course previously requiring colectomy, in most instances will remain under the care of a gastroenterologist with special expertise in IBD, therefore increasing the likelihood of SIBDCS inclusion. Moreover, the SIBDCS is not fully population-based, with a tendency towards a higher fraction of patients being treated in tertiary referral centres. On the other hand, $80 \%$ of admitted IBD patients were included by gastroenterologists working in hospitals and $20 \%$ from gastroenterologists in private practices. Whereas in the latter, mild-moderate cases presumably with intrinsically lower risk of colectomy predominate, we cannot exclude that differences in the timely initiation and escalation of medical treatment may influence colectomy rates across treating physicians. The overall colectomy rates derived in our cohort may even have been overestimated as a net effect of the aforementioned factors, and thus may be even lower in the entire Swiss IBD population.

Our study also has several strengths. Our results found on a relatively long observational time and disease duration, with a range of 0-50 years. Moreover, the SIBDCS follows a rigid and wellstructured prospective data acquisition, with yearly follow-up by physician- as well as patient-based data acquisition, in contrast to several other studies on this topic which are largely or exclusively based on retrospective data acquisition.

In conclusion, we identified overall colectomy rates to be lower than expected based on clinical experience as well as studies from recent decades, including virtually all those described in the more recent literature. Our data suggest a biphasic disease course, with an initial increase of colectomy rates within 10 years after diagnosis and subsequent decline in colectomy risk after 15 years. Colectomy risk was lowest 20-30 years after diagnosis. We found male sex, family history, pancolitis, younger age, and presence of EIM to be significant risk factors for colectomy, whereas no clear protective effect of smoking was identified. Furthermore, colectomy rates significantly decreased in Swiss UC patients, and the overall lowest colectomy rates were observed in the last 3 years of observation between 20132015. These findings are reassuring for UC patients and indicate an increase in the overall efficacy of medical treatment in recent years.

\section{Funding}

This work was supported by grants from the Swiss National Science Foundation [SNF] to the Swiss IBD Cohort [Grant No. 3347CO-108792].

\section{Conflict of Interest}

The authors declare that they have no conflict of interest.

\section{Author Contributions}

LP was involved in the concept and design of the study, analysis, interpretation of data, and was responsible for drafting the manuscript; NF was involved in the statistical analysis, interpretation of data, and drafting the 
manuscript; JZ was involved in interpretation of data and critically revising the manuscript; MS was involved in critically revising the manuscript; TG and PS were involved in revising and editing the manuscript; BM was involved in interpretation of data and critically revising the manuscript; AMS and ES were involved in interpretation of data and revising the maunscript; SRV was involved in interpretation of statistical analysis and critically revising the manuscript; GR was responsible for study design and writing up the first draft of the paper. LB was involved in the concept and design of the study, interpretation of data, and drafting and revising the manuscript. All authors read and approved the final manuscript.

\section{References}

1. Burisch J, Munkholm P. The epidemiology of inflammatory bowel disease. Scand J Gastroenterol 2015;50:942-51.

2. Hefti MM, Chessin DB, Harpaz NH, Steinhagen RM, Ullman TA. Severity of inflammation as a predictor of colectomy in patients with chronic ulcerative colitis. Dis Colon Rectum 2009;52:193-7.

3. Levine JS, Burakoff R. Extraintestinal manifestations of inflammatory bowel disease. Gastroenterol Hepatol [N Y] 2011;7:235-41.

4. Safroneeva E, Vavricka SR, Fournier N, Straumann A, Rogler G, Schoepfer AM. Prevalence and risk factors for therapy escalation in ulcerative colitis in the Swiss IBD cohort study. Inflamm Bowel Dis 2015;21:1348-58.

5. Sulz MC, Siebert U, Arvandi M, et al.; Swiss IBD Cohort Study Group. Predictors for hospitalization and outpatient visits in patients with inflammatory bowel disease: results from the Swiss Inflammatory Bowel Disease Cohort Study. Eur J Gastroenterol Hepatol 2013;25:790-7.

6. Monstada I, Hovde O, Solberga IC, Mouma BA. Clinical course and prognosis in ulcerative colitis: results from population-based and observational studies. Ann Gastroenterol 2014;27:95-104.

7. Kaplan GG, Seow CH, Ghosh S, et al. Decreasing colectomy rates for ulcerative colitis: a population-based time trend study. Am J Gastroenterol 2012;107:1879-87.

8. Leijonmarck CE, Persson PG, Hellers G. Factors affecting colectomy rate in ulcerative colitis: an epidemiologic study. Gut 1990;31:329-33.

9. Langholz E, Munkholm P, Davidsen M, Binder V. Course of ulcerative colitis: analysis of changes in disease activity over years. Gastroenterology 1994;107:3-11.

10. Hoie O, Wolters FL, Riis L, et al.; European Collaborative Study Group of Inflammatory Bowel Disease. Low colectomy rates in ulcerative colitis in an unselected European cohort followed for 10 years. Gastroenterology 2007;132:507-15

11. Moum B, Ekbom A, Vatn MH, et al. Clinical course during the 1st year after diagnosis in ulcerative colitis and Crohn's disease. Results of a large, prospective population-based study in southeastern Norway, 1990-93. Scand J Gastroenterol 1997;32:1005-12.

12. Henriksen M, Jahnsen J, Lygren I, et al.; IBSEN Study Group. Ulcerative colitis and clinical course: results of a 5-year population-based follow-up study [the IBSEN study]. Inflamm Bowel Dis 2006;12:543-50.

13. Solberg IC, Lygren I, Jahnsen J, et al.; IBSEN Study Group. Clinical course during the first 10 years of ulcerative colitis: results from a population-based inception cohort [IBSEN Study]. Scand J Gastroenterol 2009;44:431-40

14. Targownik LE, Singh H, Nugent Z, Bernstein CN. The epidemiology of colectomy in ulcerative colitis: results from a population-based cohort. Am J Gastroenterol 2012;107:1228-35.

15. Jess T, Riis L, Vind I, et al. Changes in clinical characteristics, course, and prognosis of inflammatory bowel disease during the last 5 decades: a population-based study from Copenhagen, Denmark. Inflamm Bowel Dis 2007;13:481-9.

16. Beaugerie L, Massot N, Carbonnel F, Cattan S, Gendre JP, Cosnes J. Impact of cessation of smoking on the course of ulcerative colitis. Am J Gastroenterol 2001;96:2113-6.
17. Höie O, Wolters F, Riis L, et al.; European Collaborative Study Group of Inflammatory Bowel Disease [EC-IBD]. Ulcerative colitis: patient characteristics may predict 10-yr disease recurrence in a European-wide population-based cohort. Am J Gastroenterol 2007;102:1692-701.

18. Lakatos PL, Szamosi T, Lakatos L. Smoking in inflammatory bowel diseases: good, bad or ugly? World J Gastroenterol 2007;13:6134-9.

19. Fraga XF, Vergara M, Medina C, Casellas F, Bermejo B, Malagelada JR. Effects of smoking on the presentation and clinical course of inflammatory bowel disease. Eur J Gastroenterol Hepatol 1997;9:683-7.

20. Odes HS, Fich A, Reif $S$, et al. Effects of current cigarette smoking on clinical course of Crohn's disease and ulcerative colitis. Dig Dis Sci 2001;46:1717-21.

21. Aldhous MC, Drummond HE, Anderson N, et al. Smoking habit and load influence age at diagnosis and disease extent in ulcerative colitis. Am J Gastroenterol 2007;102:589-97.

22. Ananthakrishnan AN, Issa M, Beaulieu DB, et al. History of medical hospitalization predicts future need for colectomy in patients with ulcerative colitis. Inflamm Bowel Dis 2009;15:176-81.

23. Dias CC, Rodrigues PP, da Costa-Pereira A, Magro F. Clinical predictors of colectomy in patients with ulcerative colitis: systematic review and metaanalysis of cohort studies. J Crohns Colitis 2015;9:156-63.

24. Soetikno RM, Lin OS, Heidenreich PA, Young HS, Blackstone MO. Increased risk of colorectal neoplasia in patients with primary sclerosing cholangitis and ulcerative colitis: a meta-analysis. Gastrointest Endosc 2002;56:48-54.

25. Childers RE, Eluri S, Vazquez C, Weise RM, Bayless TM, Hutfless S. Family history of inflammatory bowel disease among patients with ulcerative colitis: a systematic review and meta-analysis. J Crohns Colitis 2014;8:1480-97.

26. Henriksen M, Jahnsen J, Lygren I, Vatn MH, Moum B; IBSEN Study Group. Are there any differences in phenotype or disease course between familial and sporadic cases of inflammatory bowel disease? Results of a population-based follow-up study. Am J Gastroenterol 2007;102:1955-63.

27. Pittet V, Juillerat P, Mottet C, et al.; Swiss IBD Cohort Study Group. Cohort profile: the Swiss Inflammatory Bowel Disease Cohort Study [SIBDCS]. Int J Epidemiol 2009;38:922-31.

28. Silverberg MS, Satsangi J, Ahmad T, et al. Toward an integrated clinical, molecular and serological classification of inflammatory bowel disease: report of a Working Party of the 2005 Montreal World Congress of Gastroenterology. Can J Gastroenterol 2005;19[Suppl A]:5A-36A.

29. Jakobovits SL, Jewell DP, Travis SP. Infliximab for the treatment of ulcerative colitis: outcomes in Oxford from 2000 to 2006. Aliment Pharmacol Ther 2007;25:1055-60.

30. Gower-Rousseau C, Dauchet L, Vernier-Massouille G, et al. The natural history of pediatric ulcerative colitis: a population-based cohort study. Am J Gastroenterol 2009;104:2080-8.

31. Goudet P, Dozois RR, Kelly KA, Ilstrup DM, Phillips SF. Characteristics and evolution of extraintestinal manifestations associated with ulcerative colitis after proctocolectomy. Dig Surg 2001;18:51-5.

32. Duricova D, Leroyer A, Savoye G, et al.; EPIMAD Group. Extra-intestinal manifestations at diagnosis in paediatric- and elderly-onset ulcerative colitis are associated with a more severe disease outcome: a population-based study. J Crohns Colitis 2017;11:1326-34.

33. Lunney PC, Leong RW. Review article: Ulcerative colitis, smoking and nicotine therapy. Aliment Pharmacol Ther 2012;36:997-1008.

34. To N, Ford AC, Gracie DJ. Systematic review with meta-analysis: the effect of tobacco smoking on the natural history of ulcerative colitis. Aliment Pharmacol Ther 2016;44:117-26.

35. Höie O, Schouten LJ, Wolters FL, et al.; European Collaborative Study Group of Inflammatory Bowel Disease [EC-IBD]. Ulcerative colitis: no rise in mortality in a European-wide population based cohort 10 years after diagnosis. Gut 2007;56:497-503. 